\title{
Un análisis microeconómico de los efectos de la innovación en el desarrollo y el bienestar social
}

A microeconomic analysis of the effects of innovation in development and social welfare

\section{Resumen}

El objetivo general de este ensayo es analizar los efectos de la innovación en el crecimiento, el bienestar social y la distribución del ingreso desde la perspectiva de dos enfoques de la teoría microeconómica: La micro tradicional y la micro pos keynesiana. Las preguntas clave que deseamos contestar son: ¿cuál es el efecto microeconómico de la innovación en la empresa, en la industria y en el desarrollo económico de su entorno? ¿cuál es el razonamiento microeconómico de los efectos de la innovación en el crecimiento de la empresa, en el bienestar social? y en particular ¿cuál es el efecto de la innovación sobre la distribución del ingreso? Luego del largo olvido de la obra de J. Schumpeter y su énfasis en la innovación y el desarrollo, en las últimas décadas ha ocurrido un resurgimiento de este tema en el campo de la economía, la microeconomía y la política económica. Sin embargo, se asume de forma automática que la innovación conduce al crecimiento, al desarrollo económico y al bienestar social. En este ensayo nos queremos preguntar si esto es así o si las innovaciones agudizan los procesos de concentración productiva y del ingreso, lo cual cuestionaría aquella relación lineal, de innovación y bienestar social. Iniciamos definiendo el concepto de innovación como lo hizo J. Schumpeter, continuamos analizando la forma en cómo los clásicos y la teoría neoclásica lo incorpora en su análisis, tanto para el corto como para el largo plazo, posteriormente abordamos el análisis microeconómico heterodoxo en su versión pos keynesiana, destacando las aportaciones de A. Eichner, S. Labini entre otros, finalizando con un breve análisis comparativo de los puntos observados por esos enfoques.

Palabras clave:

- Microeconomía

- Comportamiento de la empresa

- Distribución

\section{Abstract}

Gustavo Vargas

Carlos M. Rodríguez*

The overall objective of this paper is to analyze the effects of innovation on growth, welfare and income distribution from the perspective of two approaches to microeconomic theory: The traditional and post Keynesian microeconomics. The key questions we want to answer are: What is the microeconomic effect of innovation in the firm, industry and economic development of their environment? What is the theoretical microeconomic reasoning effect of innovation in the company's growth in social welfare? and in particular, what is the effect of innovation on income distribution? After long neglect of the work of J. Schumpeter and his emphasis on innovation and development, in recent decades there has been a resurgence of this in the field of economics, microeconomics and economic policy. However, sometimes we automatically assume that innovation leads to growth, economic development and social welfare. In this paper we want to ask whether this is real, or in change, the innovations exacerbate productive processes and income concentration, in which case, we would question if the linear relationship between innovation and social welfare is fulfilled. We begin by defining the concept of innovation as did J. Schumpeter, we continue to analyze the way how the classical and neoclassical theory incorporates it into his analysis, both for the short and long term, then boarded the heterodox microeconomic analysis in its post- Keynesian version, highlighting the contributions of A. Eichner, S. Labini among others, ending with a brief comparative analysis of the points raised by the approaches.

\section{Keywords: \\ - Microeconomics \\ - Firm Behavior \\ - Distribution}

JEL: B21, D21, D3

\section{Introducción}

Joseph Schumpeter propuso la tesis de que la innovación es la fuerza motriz del desarrollo económico, con lo cual abrió una nueva era en la teoría económica donde la innovación se convirtió en el objeto de estudios de diferentes teorías y en un instrumento de la política económica para lograr el crecimiento. 
Previamente los economistas clásicos, como Adam Smith, David Ricardo y Carlos Marx ya habían abordado las diferentes formas de innovación, por ejemplo A. Smith explica la creación y aumento de la riqueza gracias a la innovación organizacional en la forma de una nueva división del trabajo. El mismo Marx estudió el impacto de la introducción de las innovaciones en la industria textil y manufacturera en la época del desarrollo temprano del capitalismo, sin embargo, fue Schumpeter quien abordó de forma sistemática el fenómeno de la innovación como el mecanismo que conduce al desarrollo económico. De ahí su gran relevancia contemporánea.

J. Schumpeter acuñó el concepto de innovación para identificar las nuevas combinaciones de los recursos productivos, los cuales cubren cinco casos: La introducción de un nuevo bien o la mejora en su calidad, la introducción de un nuevo método de producción, la apertura de un nuevo mercado, la conquista de una nueva fuente de aprovisionamiento de materias primas o de bienes semimanufacturados, y la creación de una nueva organización de cualquier industria, como la de una posición de monopolio o bien la anulación de una posición de monopolio que había existido con anterioridad (Schumpeter, 1912). El resultado de una innovación exitosa en términos económicos es para la empresa una ganancia incrementada, a la cual le llama ganancia de la innovación. Esta ganancia es la motivación para que los empresarios y las empresas busquen e introduzcan las innovaciones. El resultado de este cambio por el emprendedor y el efecto de seguimiento de las demás empresas desencadena un proceso que acelera los intercambios y la producción que finalmente resulta en el crecimiento económico (Schumpeter, 1934).

Diferentes economistas han continuado estudiando la innovación desde diferentes enfoques, dos enfoques contrastan en su análisis y conclusiones, en particular con relación a la innovación y su efecto sobre el crecimiento económico: la escuela neoclásica y la poskeynesiana, las cuales analizamos a continuación.

\section{Microeconomía neoclásica y la innovación}

En esta teoría podemos encontrar dos escenarios en los que se explica los efectos de la innovación, en competencia perfecta y la imperfecta (monopolio). En ambos casos las conclusiones son similares. El mercado cumple una función fundamental: determina los precios y las cantidades de equilibrio, los cuales en el largo plazo garantizan que se logre el bienestar social óptimo. Por tanto el precio, que es la variable que buscamos analizar está determinada por las libres fuerzas de la oferta y la demanda, dicho precio se modificara en el corto plazo 
Economía Informa núm. 383 noviembre - diciembre • 2013 | " " "

por cambios en cualquiera de esos factores. Pero en el largo plazo su comportamiento tiene que ver con los cambios en el lado de la oferta y en particular en la tecnología que está implícita en las condiciones de la oferta. Por lo que el estudio de las innovaciones y su efecto lo orientamos al lado de la oferta.

La elaboración teórica de partida para la construcción de la teoría de la oferta, la cual sirve como base tanto para los modelos de competencia perfecta como imperfecta, es la definición de la función de producción. Ésta la han definido como una función matemática que especifica la producción máxima que se puede producir con una cantidad determinada de insumos. Se supone que ésta función refleja los conocimientos y el estado de la tecnología y la ingeniería correspondiente a la actividad productiva. (Samuelson y Nordhaus, 2010, Varian, Vargas). Un segundo supuesto es el que los factores o insumos de la producción, en particular el trabajo y el capital, son sustitutos, lo cual les permite construir las conocidas curvas de isocostos, las cuales en tangencia con la línea isocuanta determina el equilibrio de la empresa, en el uso del capital y el trabajo. En el corto plazo, el precio está sujeto a movimientos temporales de oferta y de demanda, y la tecnología se mantiene constante y es sólo en el largo plazo donde se admiten los cambios de carácter tecnológico.

Un desarrollo tecnológico (una innovación de tecnología) en primer lugar desplaza hacia arriba la función de producción elevando la producción y la productividad del trabajo y del capital, al mismo tiempo que desplaza hacia abajo la isocuanta de producción. Como afirma A. Kutsoyianis, la mejora tecnológica se expresa en que "se puede obtener el mismo volumen de producción con menos insumos de factores, o un mayor volumen de producción con los mismos insumos." (A. Kutsoyiannis, 1988, p. 95) El análisis de la innovación se puede realizar en dos escenarios una de competencia perfecta y el segundo de competencia imperfecta, los cuales presentamos en dicho orden.

\section{Competencia perfecta e innovación}

Bajo el escenario y los supuestos de competencia perfecta definidos por la teoría neoclásica, el precio de equilibrio se determina en el mercado por la interacción de las curvas de la oferta y la demanda. Una innovación tecnológica generará un desplazamiento de los costos medios, lo que determina un traslado de los costos marginales hacia abajo. Dados los supuestos de libre flujo de información y capitales, tendrá lugar un proceso automático de difusión de la tecnología, por lo que todas las empresas serán capaces de introducir las mismas innovaciones y producir a menores costos. El resultado final es que los precios se reducen al tiempo que las cantidades producidas aumentan. 
El resultado de una innovación es el desplazamiento de la curva de la oferta de la empresa en competencia perfecta hacia la derecha, iniciando un desplazamiento del equilibrio de corto plazo hacia una trayectoria de largo plazo. Las innovaciones se acompañan de un mecanismo de difusión automático, con lo cual lo que ocurre en el mercado es un proceso de nuevas inversiones hacia las actividades más rentables (por la innovación) lo cual se traduce en una reducción del precio y a un aumento de la cantidad producida en todo el mercado. El resultado es un mejoramiento en el estado de bienestar social general.

\section{Monopolio e innovación}

En la teoría neoclásica, aún en el caso del monopolio, dadas las características tecnológicas ya definidas, una innovación da lugar a un desplazamiento de la función de producción, lo cual permite a la empresa producir la misma cantidad con menos insumos. Esto provocará que sus costos medios y marginales disminuyan, y su productividad aumente.

Como tenemos solo una empresa, ésta enfrenta la demanda total del mercado, por lo que el punto de equilibrio del monopolio se determina, dado que el comportamiento de productor se asume como racional en el sentido optimizador, donde el ingreso marginal es igual al costo marginal maximizando sus beneficios. (Pindyck y Rubinfeld, 2001, p. 338),

En segundo lugar, cuando se presenta una innovación, no existe efecto difusión pues solo existe una empresa, el monopolio que en este caso sería un "monopolio innovador" se queda con el conocimiento de la innovación. La innovación en el caso de que sea de tecnología, de mercado o de organización, da lugar una reducción de costos, el ingreso marginal no se modifica y el punto de equilibrio se encuentra en un nivel inferior al equilibrio anterior, lo cual determina que el precio baje y la cantidad producida aumente. En este nuevo punto de equilibrio, si bien la producción aumenta y los precios bajan son aún superiores a los correspondientes a la competencia perfecta.

En síntesis, en el caso del monopolio los resultados son: por una parte, un aumento de la productividad total, de la productividad del trabajo y con este, del salario de los trabajadores; y por otra, la innovación se traduce en menores precios y aumento de la cantidad producida, por lo que se garantiza que el bienestar social aumente. 
Economía Informa núm. 383 noviembre - diciembre • 2013 | " " "

\section{Teoría microeconómica Pos Keynesiana y la innovación}

La teoría microeconómica de la oferta en la escuela pos keynesiana (PK) presenta una visión que contrasta con la visión neoclásica tanto en su discurso analítico como en sus resultados. Si bien ambas teorías coinciden en que la innovación es uno de los mecanismos más importantes que explican el crecimiento económico, las diferencias se presentan en el aparato conceptual de análisis y en consecuencia, en las conclusiones sobre los efectos de la innovación. Por lo cual a continuación abordamos en primer lugar algunos elementos fundamentales para comprender el marco teórico microeconómico de la teoría pos keynesiana, (su enfoque de empresa, precios y costos, margen de ganancias e inversión) y en segundo lugar, se analizan los efectos de las innovaciones (en cuanto a su efecto en la empresa, en la industria y la distribución del ingreso). Dentro de esta escuela, en esta oportunidad vamos a analizar las aportaciones de: A. Eichner, Harcourt, Kenyon y Sylos Labini entre otros.

\section{Enfoque de empresa}

En el enfoque PK, la empresa no es un actor pasivo dentro del mercado en el cual opera. Existe una relación de efecto y causa en un proceso complejo que evoluciona en el tiempo, el cual modifica tanto a las empresas como a los consumidores. La empresa es por tanto el actor económico fundamental cuyas acciones afectan directamente el desenvolvimiento de la economía.

Para A. Eichner la empresa relevante es la megacorporación la cual define como una organización compleja que está en continuo crecimiento y tiene poder para determinar sus precios. El mercado no es un espacio homogéneo donde los agentes participen en igualdad de circunstancias y con el mismo poder e influencia. El mercado de producción capitalista está dominado por esas grandes empresas o corporaciones que tienen la cualidad y capacidad que solo ellas poseen, que consiste en ser organizaciones independientes tomadoras de decisiones, las cuales influyen y afectan a las otras empresas, a los consumidores y a las instituciones.

La megacorporación es un concepto que captura la esencia del sistema económico actual consistente predominantemente de organizaciones oligopólicas que dominan la industria, con la resultante habilidad de fijar un precio utilizando un mark up (margen) sobre sus costos, y con la capacidad de gestionar los procesos de financiamiento de la empresa para su expansión y crecimiento constante (Eichner, 1988, p. 29). 
Por su parte Sylos Labini, utiliza el concepto de oligopolio para caracterizar a la empresa capitalista dominante en una industria, donde existen pocas grandes empresas con capacidad para controlar el mercado. Sin embargo, este autor muestra que en el mercado coexisten junto a los oligopolios, medianas y pequeñas empresas que también participan en la industria, pero que tienen, en general, un papel menos relevante en términos de fijación de precios, aunque son importantes para determinar un precio en el mercado al conformar parte de un conjunto de empresas que participan en la determinación del precio. Si bien las empresas de menor tamaño producen a mayores costos, generan una producción, la cual al conjugarse con la producción de la gran empresa, la cantidad producida por ellas es relevante en la determinación de un precio de equilibrio del mercado. En este escenario de interacción de empresas de diversos tamaños, si una empresa intentara entrar, alteraría el precio, de manera que su entrada no sería factible en términos de costos, y alteraría el nivel de precios.

Para F. Lee (1988) en un enfoque más amplio de la heterodoxia, la empresa es una unidad de producción de bienes y servicios, con el fin de venderse para obtener una ganancia. Dichos bienes deben tener la cualidad de satisfacer las necesidades de los consumidores, pero el objetivo de la empresa no es satisfacer las necesidades de los compradores, sino obtener ganancias. Así, las empresas construyen y desarrollan estrategias de todo tipo para vender sus productos, crecer, y dominar su mercado. En este sentido M. Lavoie enfatiza la importancia del poder de mercado (Lavoie, 2005).

En esta escuela las empresas poseen al menos cinco características: a) son unidades de producción, b) son entidades históricas, c) son organizaciones para la producción, d) son instituciones, y finalmente, e) son espacios de conflicto donde se determina la distribución del ingreso. Estas empresas tienen la cualidad de determinar sus precios a través de un Mark-up así como sus estrategias de inversión.

En síntesis para los pos keynesianos, el corazón del sistema capitalista está en la empresa y su capacidad de decisión para fijar precios y estrategias de expansión. El análisis relevante se orienta al mercado, considerando la dominancia de las grandes empresas u oligopolios, un mercado donde no todos los agentes son homogéneos, sino que el mercado está formado por empresas de diferentes tamaños, con capacidades distintas para determinar la dirección de los precios y de la industria en su conjunto. La empresa por tanto consiste en una organización dinámica sujeta a una ley de expansión y crecimiento. La pareja de precios y cantidades son solo una fotografía en un instante que puede o no volverse a 
Economía Informa núm. 383 noviembre - diciembre • 2013 | " " "

repetir en cuanto a magnitud pero no en su dimensión histórica la cual corresponde a una sola observación en la sucesión de datos en el proceso de la producción. Los precios y las cantidades son relevantes en cuanto al papel que tienen en el proceso de creación y distribución de ingresos con el objetivo final de crecer. En este sentido, el análisis micro de la oferta se divide en dos partes: la determinación del precio y la cantidad vendida, y en segundo el análisis de expansión.

Tecnología, fijación de precios y los costos

La tecnología definida por la escuela pos keynesiana está caracterizada por que la producción está dominada por un conjunto de recursos productivos los cuales son complementarios y en el tiempo reportan rendimientos crecientes. Esto determina que en la zona relevante en la cual opera la empresa, los costos variables medios y los costos medios totales se aproximen a una horizontal y, en consecuencia, los costos marginales son irrelevantes en la toma de decisiones de la empresa (Andrews, 1959; Eichner, 1988; Lavoie, 1992; Vargas, 2006).

El procedimiento de fijación de precios está determinado por dos factores, en primer lugar la estimación del precio a partir de los costos de la empresa más un margen de ganancia o Mark-up, por tanto, los precios se fijan, en general por la regla de agregar un margen de beneficio bruto GPM a los costos variable medios AvC.

$$
\mathrm{P}=\mathrm{AVC}+\mathrm{GPM}
$$

El margen bruto de beneficio es igual al costo fijo promedio AFC, más el margen de beneficio neto NPM.

$$
\mathrm{GPM}=\mathrm{AFC}+\mathrm{NPM}
$$

La suma del AVC, AFC y NPM expresa la composición de los precios, los cuales son fijados por la empresa considerando cubrir, no solo todos sus costos, sino también un beneficio normal o esperado. En otras palabras, se toma como base el coste directo unitario AVC, se añade un porcentaje para cubrir el coste fijo y un ulterior tanto porciento convencional (Lee, 1988; Lavoie, 1992; Hall y Hitch, 1939).

Dado que la empresa no opera a plena capacidad, buscará continuamente aumentar la utilización de la misma, es decir aumentar sus ventas, pero estas estarán determinado por su fuerza de ventas, la demanda efectiva del mercado 
y su expansión. Claramente no existe un punto de equilibrio que sea estable y duradero, tanto por las estrategias de ventas de la empresa, como de sus rivales y el comportamiento de los compradores.

El segundo conjunto de factores que influyen en los precios a través del margen de ganancia es el estado de la competencia de su mercado, donde juegan las barreras a la entrada, el efecto sustitución, la amenaza de entrada por otros competidores, las innovaciones, y las posibilidades de intervención del Estado.

Así, a pesar de que la gran empresa tiene cierta capacidad para influir en el precio, ésta decisión estará acotada por variables tales como: La extensión absoluta del mercado, la elasticidad de la demanda, las diversas tecnologías, los precios de los factores variables y de las máquinas que intervienen, junto con las tecnologías, en la determinación del coste medio total de las empresas. En particular se debe mencionar que son "las reacciones de los rivales, efectivos o potenciales, mucho más que las de los consumidores, las que influyen en la conducta de los hombres de negocios" (Sylos, 1966, p. 67). El temor a que grandes empresas intenten ingresar al mercado incentivadas por un margen de ganancia muy alto, restringe el poder de la gran empresa de manipular el precio. Un elemento adicional, pero de especial importancia que influye en el nivel y variación de los precios, en particular en la determinación del markup, es la necesidad de fondos adicionales de inversión de la empresa dominante, es decir sus planes de inversión y expansión (Eichner, 1988, pp. 32).

\section{Ganancia, Inversión e innovación}

En el análisis pos keynesiano la empresa existe porque busca crear ganancias, y no porque sea parte de un sistema de equilibrio. Las ganancias son el objetivo y la razón de ser de todas las empresas capitalistas. La inversión es la forma por la cual la empresa crece y se expande con el fin general de obtener un volumen mayor de ganancias. Si la tecnología no se modificara al igual que el estado de la competencia, el margen de ganancia podría permanecer constante, sin embargo, una innovación abre las posibilidades de nuevas inversiones, nuevos negocios, o hace a las empresas existentes aún más rentables. Las innovaciones le ofrecen a las empresas que las introducen una ventaja sobre sus rivales que puede dar lugar a una ganancia de innovación aumentando el margen de ganancia previo y coloca a la empresa en una trayectoria de crecimiento e innovación.

Habiendo definido a la ganancia como el motivo principal de la empresa, y recordando que la gran corporación fija sus precios gracias a su poder monopólico, se pueden identificar dos componentes de la ganancia: una parte es la 
Economía Informa núm. 383 noviembre - diciembre • 2013 | " " "

que se utiliza para el pago de dividendos y el pago del desgaste del capital fijo, y otra la más importante en términos teóricos es la destinada al crecimiento, por lo que el precio y sus variaciones están influidos por "la necesidad de fondos de inversión para expandir su capacidad dentro de la industria en línea con el crecimiento de las ventas" (Eichner, 1988, p. 33).

Los planes de inversión entran en la determinación del margen de ganancias, del precio y de sus variaciones. Si bien las inversiones pueden estar en parte financiadas por créditos bancarios, la fuente más importante es el flujo de efectivo generado en la empresa por sus operaciones corrientes. "Los planes de inversión y el tamaño del margen están inexorablemente ligados por medio de la demanda y oferta de fondos en forma de ganancias retenidas con las cuales la empresa financia los proyectos de inversión propuestos. ... el precio efectivo se determina directamente mucho menos por la demanda corriente que por el margen que la empresa considera necesario para aumentar su capacidad en medida suficiente para satisfacer el futuro nivel de demanda esperado" (Harcourt y Kenyon, 1988, pp. 229-230).

En la gran empresa, en particular aquellas que son innovadoras, la planeación de la utilización del flujo de efectivo de la empresa asigna una partida al desarrollo de sus productos, procesos tecnológicos, de organización o de mercados, o bien compra estas innovaciones a proveedores especializados. Ya sea que la empresa cuente con departamentos de innovación y desarrollo o que adquiera las innovaciones en el mercado, el flujo de efectivo tiene un papel central en su financiamiento. En términos dinámicos lo importante es que una innovación exitosa, por ejemplo de proceso, dará lugar a un ciclo virtuoso que aumentará el flujo de efectivo y con esto el financiamiento de inversiones en innovaciones.

Innovación, tecnología, costos, precios, y margen de ganancia

Si consideramos el caso de una industria oligopólica donde una empresa es la líder, la cual introduce una innovación que reduzca costos o una tecnología ahorradora de trabajo, entonces la curva de costos variables medios se desplaza hacia abajo, los costos medios exante no lo sabemos, pero si suponemos un cambio tecnológico en la producción que no modifica sustancialmente el bien final o que genere un bien similar al previo a la innovación, entonces, en efecto, los costos medios totales si se reducen y la curva de costos medios totales se desplaza hacia abajo. En esta perspectiva los precios fijados por los oligopolios no tienen porque reducirse, la situación "normal" en el capitalismo 
contemporáneo es que se mantengan constantes y en muchos casos aumentan. Esta rigidez de los precios a la baja, con costos menores por la innovación va a determinar que el margen de ganancia media y la total aumenta.

En este caso, la innovación tiene un efecto positivo sobre los costos, con un consecuente crecimiento en el margen de beneficios, y se puede dar también lugar a un incremento en la demanda, pero este incremento se deberá explicar sobre todo por cuan sensibles sean los consumidores a la nueva publicidad asociada a los nuevos bienes producidos con esta nueva tecnología. En cuanto a los precios, como estos no están determinados por la curva de demanda (que en la práctica es desconocida), no se modifican salvo por las estrategias de competencia de las empresas.

El anterior es un caso de innovación tecnológica sobre la producción de un bien ya conocido, pero que también puede aplicarse muy bien a una innovación organizacional o de mercado, en este último caso la curva de los costos medios, también se desplazaría hacia abajo y a la derecha dando lugar a un volumen mayor de ganancias. En el caso de innovación y diferenciación de productos es un poco más elaborada pero sigue el mismo patrón de comportamiento.

En el caso de una innovación tecnológica de producto que da lugar a una diferenciación del mismo, podemos observar casos en que los nuevos productos reportan costos mayores, pero que el poder del oligopolio y su conocimiento del mercado le permite fijar un margen más alto de ganancias y si por la diferenciación las ventas de los nuevos productos aumentan, las ganancias y el desempeño de las empresas serían mayores gracias a la innovación.

Innovación, bienestar social y distribución del ingreso

En términos de bienestar y distribución del ingreso, los resultados de la innovación en la economía contemporánea son muy diferentes a los que pudieron observarse en el periodo de formación del capitalismo y en los modelos teóricos de competencia perfecta. En el marco de los mercados dominados por las grandes corporaciones y las industrias dominadas por oligopolios, las innovaciones, si bien generan crecimiento económico, no se traducen en bienestar social y mejores niveles de distribución del ingreso.

En el caso mencionado de innovación en los procesos de producción, o variación de los coeficientes técnicos ya existentes, se pueden identificar los siguientes efectos: Al interior de la empresa el aumento de la productividad de los factores, dado un volumen de ventas, puede dar lugar a la contrata- 
Economía Informa núm. 383 noviembre - diciembre • 2013 | " " "

ción de menos trabajadores, lo cual reduce el costo medio por unidad. Si el volumen de ventas aumenta, el número de trabajadores puede permanecer constante o también aumentar si la tecnología y la capacidad aumentan. En cualquier caso la cantidad de trabajo requerida por unidad de producto habrá disminuido. El costo variable medio se habría reducido (Eichner, 1988; Lee, Lavoie, 2005; S. Labini, 1966, p. 157).

El salario de los trabajadores que permanecen en la producción, no aumenta de forma automática, sino que está sujeta a un nuevo proceso de negociación salarial, el cual puede ser de forma individual o a través de sus sindicatos. En este último caso el crecimiento de los salarios dependerá de forma natural de la autonomía y del poder de los mismos. Esto dará como resultado lo que en la escuela pos keynesiana se conoce como el conflicto distributivo.

En segundo lugar, en el marco oligopólico el efecto de difusión esta disminuido, no solo por factores institucionales como los derechos de propiedad y patentes, sino por la naturaleza misma de las empresas y la industria. El poder de la empresa de fijar el precio le permite no bajarlo, además porque no tiene la presión de la competencia. En una industria oligopólica, la innovación y sus efectos positivos sobre costos no se difunden como en competencia perfecta, debido a la capacidad de la gran empresa de retener la innovación y la información relevante, y cuando esto ocurre las otras empresas oligopólicas tampoco trasladan la reducción de costos a los precios. Esto se traduce en un amento del margen de beneficio para la empresa innovadora y posteriormente para las seguidoras.

En tercer lugar, el mecanismo de crecimiento que se supone de forma automática ocurre con las innovaciones en mercados con muchos productores y flujo de capitales, se ve disminuido. En una economía dominada por los oligopolios, con procesos continuos de innovación tecnológica, el trabajo no es reabsorbido como en el caso de la competencia perfecta. La explicación está en que la innovación no se traduce en menores costos para toda la industria, debido a que no se difunde libre ni automáticamente la innovación, y por tanto no hay menores precios, los cuales generarían un aumento del poder adquisitivo del mercado reduciendo la capacidad de absorción y crecimiento de la demanda efectiva. El mecanismo de precios no opera, y el efecto del incremento en el ingreso relativo no tiene lugar o se reduce y con ello la consecuente necesidad de más producción que reabsorba a la fuerza de trabajo primeramente expulsada ya no opera o se ve claramente mermada. Así, aparece una tendencia en el largo plazo a una reducción de la tasa de crecimiento de la economía y del desempleo estructural. 


\section{Conclusiones}

El progreso técnico es un elemento importante que forma parte de las fuerzas del desarrollo. Abre la oportunidad a la generación de beneficios extraordinarios que visto desde distintas corrientes de pensamiento pueden generalizarse o bien, mantenerse en unas cuantas manos. Los distintos cuadros analíticos brindados por las escuelas nos permiten observar cuáles son los efectos de una innovación mediante supuestos determinados, acercándonos a un mejor entendimiento de lo que sucede en una economía al introducirse una innovación.

Los marcos analíticos están sustentados en el conjunto de supuestos que le dan coherencia a la teoría, lo interesante es, cuál de esos supuestos son aquellos que más se acercan a la realidad y por tanto nos permiten explicarla de mejor manera. La pregunta de cuáles son los efectos esperados de la introducción de una o un conjunto de innovaciones en el crecimiento y la innovación desde una perspectiva del análisis microeconómico depende de la teoría que se utilice, de sus supuestos y el conjunto de relaciones teóricas.

La teoría neoclásica afirma que como efecto de la introducción de innovaciones se incrementa la producción, se reducen los precios, aumenta la productividad del trabajo, y se llega a un nuevo estado de equilibrio, el cual se acompañan de un nivel más alto de bienestar económico. Esto podrá ser cierto en un mundo dominado por la competencia perfecta, donde existen una gran cantidad de empresas que producen un mismo bien, la información y los capitales fluyen libremente. Es un conjunto de supuesto que contrastan con la realidad que se caracteriza por una sociedad dominada por oligopolios.

La teoría poskeynesiana, no se ha marcado una agenda de investigación dirigida explicar cómo llegar al equilibrio de los mercados, sino el como los mercados funcionan respetando sus características fundamentales. En una economía dominada por oligopolios y megacorporaciones, las cuales tiene capacidad para fijar precios y determinar flujos sustanciales hacia la investigación y el desarrollo, se puede demostrar que el cambio tecnológico da lugar al aumento en la producción total, la productividad del trabajo, lo cual se acompaña de reducciones en los costos, pero los precios en este contexto no bajan, lo cual se traduce en un incremento de las utilidades de la empresa innovadora, por lo que el efecto de difusión se limita, lo mismo que el impacto de crecimiento. Las ganancias de innovación no se trasladan a la sociedad en términos de más bienestar, sino que se transforman en crecientes flujos de efectivo los cuales serán utilizados para aumentar el poder de los oligopolios y para financiar sus estrategias de expansión a nivel nacional e internacional. 
Economía Informa núm. 383 noviembre - diciembre • 2013 | " " "

Las políticas de innovación y emprendedurismo para su éxito deberán considerar las nuevas condiciones de oligopolio que efectivamente dominan en la economía mexicana.

\section{Bibliografía}

Andrews, P. W. S. (1959), Economía de las empresas Industriales, Santiago Rueda Editor, Buenos Aires.

Corona, Leonel (2012), "Innovar la innovación. Evolución y perspectivas de los conceptos de innovación y emprendedor", x Congreso Nacional da la RIDIT, Toluca, México.

Eichner, Alfred (1988), "Microfundations of the Corporate Economy", en Toward a New Economics, Essays in Post-Keynesian and Institutionalist Theory, M.E. Sharpe, N.Y. cap. 3.

Harcourt, G. y Peter Kenyon (1988), "La fijación de precios y la decisión de inversión”, en, Ocampo, José Antonio (1988) “Economía Poskeynesiana” Lecturas del Trimestre Económico num. 60, FCE, México, Capítulo 9.

Koutsoyiannis, Anna (1988) Non-Price Decisions McMillan Education, Hong Kong.

Hall, R. L. y Hitch, C. J. (1939), "Price Theory and Business Behaviour" Oxford Economic Papers, os-2 (1): 12-45.

Lavoie, Marc (1992), "Foundations of Post-Keynesian Economic Analysis" Edward Elgar.

-_(2005), "La economía Postkeynesiana, un antídoto del pensamiento único" Icaria Antrazyt.

Lee, Frederich (1988), Post Keynesian Price Theory Cambridge University Press, Uk.

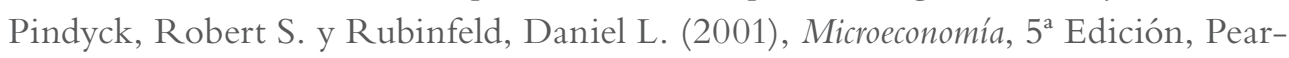
son-Prentice Hall.

Samuelson, Paul y D. Nordhaus (2010), Microeconomía con aplicaciones a Latinoamérica Decimonovena edición, Mac Graw Hill, México.

Schumpeter, Joseph A. (1912), Teoría del desenvolvimiento económico, Fondo de Cultura Económica, Primera edición en español, 1944, México D. F.

SylosLabini, P. (1966), Oligopolio y progreso técnico, Ediciones Oikos-Tau, Barcelona, España.

SylosLabini, P. (1988), Las Fuerzas del Desarrollo y del Declive, Oikos-Tau, Barcelona, España.

Varian, Hal R. (2011), Microeconomía intermedia, 8a edición, Antony Bosch Editor.

Vargas, Gustavo (2006), Teorías de la Empresa y de la Competitividad Editorial Castdel, México, D.F. 